\title{
VALORACIÓN DE PROYECTOS DE TURISMO DEPORTIVO CON OPCIONES REALES: EL CASO DE UN CAMPO DE GOLF EN LAS RÍAS BAIXAS ${ }^{1}$
}

\author{
Marcos Vizcaíno-González* \\ Soraya Sáenz-Díez Callejo \\ Universidade da Coruña
}

\section{RESUMEN}

El objetivo de este artículo es estudiar la conveniencia de la metodología de opciones reales para valorar proyectos de inversión en el sector del turismo deportivo, utilizando el caso de la construcción de un campo de golf como ejemplo para ilustrar la exposición. Tras valorar el proyecto inicial, se complementa el análisis considerando las opciones de diferir, abandonar, ampliar y reducir el proyecto. Como conclusión, se recomienda el ejercicio de la opción de diferir y, a continuación, el ejercicio de la opción de reducir. Ello supone retrasar el inicio del proyecto respecto al plan inicial, y a continuación iniciarlo con una escala más reducida, lo que implica externalizar parte de los servicios del complejo deportivo. Si bien las opciones reales constituyen una metodología de uso general, su aplicación al sector del turismo deportivo es aún limitada. Este artículo muestra la utilidad de identificar opciones de flexibilización y modularidad en el sector del turismo deportivo, dada la dimensión temporal y financiera de los proyectos, así como la incertidumbre del entorno.

Palabras clave: opciones reales; opciones financieras; valoración de proyectos; turismo deportivo.

Fecha de recepción: 17 de junio de 2017.

Fecha de aceptación: 9 de febrero de 2018.

* Departamento de Empresa, Facultad de Economía y Empresa. Universidade da Coruña. Campus de Elviña. 15071 A CORUÑA (España).E-mail: marcos.vizcaino@udc.es

1 Investigación realizada como parte de un Trabajo Fin de Grado para la obtención del Grado en Administración y Dirección de Empresas en la Facultad de Economía y Empresa de la Universidade da Coruña. 


\title{
Valuation of sports tourism projects using real options: the case of a golf course in Rías Baixas
}

\begin{abstract}
This study intends to address the convenience of a methodology based on real options to assess investment projects in the sports tourism industry, using the case of the construction of a golf course as an example to illustrate the exposition. After evaluating the initial project, the analysis is complemented by considering the options to defer, abandon, expand and reduce the project. As a conclusion, it is recommended to exercise the option to defer and then exercise the option to reduce. This means delaying the start of the project, and then starting it with a smaller scale, which implies outsourcing part of the sports complex's services. Although the real options methodology is a general-purpose approach, its application to the sports tourism industry is still limited. This article shows the usefulness of identifying flexibilization and modularity options in the sports tourism industry, given the temporal and financial dimension of its projects, and considering the uncertainty of the environment.
\end{abstract}

Keywords: real options; financial options; project valuation; sports tourism.

\section{INTRODUCCIÓN}

La metodología de opciones reales aplica el modelo binomial originalmente concebido para valorar opciones financieras a la evaluación de proyectos de inversión. Las opciones reales nacen para dar respuesta a las limitaciones de los métodos tradicionales de valoración de proyectos, ya que estos no resultan enteramente adecuados para valorar proyectos sujetos a un elevado nivel de incertidumbre y flexibilidad. No obstante, las opciones reales no se plantean como alternativa a dichos métodos tradicionales, sino como un complemento que resulta útil para llegar a una valoración del proyecto lo más acertada posible. En particular, la metodología de opciones reales permite incorporar la posibilidad de retrasar la inversión (opción de diferir), de ampliar o reducir la dimensión del proyecto (opciones de expansión y reducción, respectivamente), e incluso de abandonar el proyecto (opción de abandono). Este análisis suministra información muy útil para la toma de decisiones por parte del inversor, ya que así puede reaccionar a los posibles acontecimientos o contratiempos que puedan suceder a lo largo de la vida del proyecto (Pindado, 2012).

El objetivo principal de este artículo es aplicar la metodología de las opciones reales a un proyecto de turismo deportivo, un sector en el cual las aplicaciones de esta metodología son aún limitadas debido a que no siempre se identifican con claridad oportunidades de flexibilización y modularidad. Sin embargo, y utilizando como ejemplo el caso de un campo de golf en las Rías Baixas, se muestra el potencial de las opciones reales para valorar proyectos de inversión en este sector particular, si se identifican con precisión los escenarios de opcionalidad incorporados al proyecto. La construcción de un campo de golf es un proceso de gran complejidad, influenciado por numerosos condicionantes, que pueden modificar los parámetros económico-financieros del proyecto en cualquier momento de su desarrollo. 
La metodología de opciones reales se presenta como recurso para incorporar la flexibilidad que permita responder a esos imprevistos durante la vida del proyecto. Por ello, el artículo se centra en la metodología, mostrando con un ejemplo numérico su potencial. Por ello, se hace preciso realizar algunas simplificaciones en las condiciones iniciales del proyecto, que requerirían un análisis riguroso, lo que sobrepasa el objetivo del artículo.

El resto del artículo se estructura como sigue. La segunda sección aporta el marco teórico que contextualiza el resto de la investigación. La tercera sección describe la metodología aplicada. La cuarta sección presenta y comenta los resultados. Finalmente, la quinta sección ofrece las conclusiones del estudio.

\section{MARCO TEÓRICO}

La metodología de opciones reales hace uso de la teoría de las opciones financieras para evaluar activos físicos o reales (Ayús, Lenin y Aristizábal Velásquez, 2012; Mun, 2002). Ello quiere decir que el activo subyacente es un activo real o inversión productiva, que podrá ejecutarse o no en un futuro en función de su aportación al valor de la empresa. La metodología de opciones reales permite a los directivos tener más capacidad de reacción ante eventos que puedan afectar al valor de una inversión, aumentando así las ganancias o mitigando las pérdidas (García Machado, 2001). Los métodos tradicionales basados en el descuento de flujos de fondos son generalmente aceptados como una técnica adecuada para la valoración de un proyecto. Pero existen algunos proyectos que poseen características que no son bien recogidas por estos métodos de valoración tradicionales, debido a su elevado nivel de incertidumbre y a la necesidad de incorporar flexibilidad a la hora de tomar la decisión de inversión. Estos métodos clásicos de valoración son adecuados cuando se trata de evaluar decisiones de inversión que no pueden ser retrasadas, del tipo "ahora o nunca", o que no incorporan flexibilidad en su desarrollo (Pindado, 2012). Sin embargo, en proyectos que no encajen en esta descripción, se aconseja acudir a la metodología de opciones reales como complemento al análisis tradicional. Se pueden identificar diferentes tipos de opciones:

- Opción de diferir: esta opción consiste en retrasar la inversión en un plazo establecido, lo cual da la posibilidad de obtener información adicional antes de tomar una decisión (Pindado, 2012). Diferir será más atractivo cuando hay mucha incertidumbre y cuando los flujos de caja inmediatos sean pequeños. Proporciona al propietario de la opción el derecho a posponer su realización durante un tiempo determinado. Así pues, la opción de diferir equivale a una opción de compra americana sobre el proyecto de inversión (García Machado, 2001).

- Opción de expansión: la opción de ampliar un proyecto de inversión proporciona a su propietario el derecho a adquirir una parte adicional del mismo a cambio de un coste, que sería el precio de ejercicio (Mascareñas, 2010). Es equivalente a adquirir una opción de compra americana sobre una parte adicional del proyecto inicial. Por lo que, si las condiciones son favorables, un proyecto que pueda ampliarse tiene mayor valor que el mismo sin esa posibilidad de ampliación.

- Opción de reducción: la opción de reducir un proyecto de inversión proporciona a su propietario el derecho a renunciar a una parte del mismo a cambio de un ahorro 
adicional: el precio de ejercicio (Mascareñas, 2010). Este tipo de opción equivale a adquirir una opción de venta americana sobre una parte del proyecto inicial. Muchos proyectos pueden ser diseñados "por módulos" de tal forma que se pueda reducir la producción o el tamaño en el futuro, si las condiciones no son adecuadas (García Machado, 2001).

- Opción de abandono: esta opción proporciona a su propietario el derecho a vender, liquidar o cerrar un proyecto determinado a cambio de un precio (Mascareñas, 2010). Equivale a una opción de venta americana donde el precio de ejercicio es el valor residual del proyecto. Por lo tanto, esta opción se aplica ante condiciones de mercado desfavorables, en las cuales el inversor optaría por abandonar las operaciones permanentemente y liquidar sus activos (García Machado, 2001).

- Opción de elección: es aquella que permite escoger entre varias opciones de las anteriormente citadas. Es necesario considerar la interacción de los diferentes tipos de opciones, debido a la naturaleza mutuamente excluyente e independiente de algunas de ellas (Pindado, 2012).

La metodología de opciones reales ha sido ampliamente utilizada para la valoración de empresas y proyectos de inversión con carácter general (Abel, Dixit, Eberly y Pindyck, 1996; Amram y Kulatilaka, 1999; Bowman y Hurry, 1993; Brennan y Schwartz, 1985; Copeland Thomas, Koller y Murrin, 1994; Copeland y Antikarov, 2001; Dixit, 1994; González-Echeverri, Mora-Valencia y Solano, 2015; Koller, Goedhart y Wessels, 2010; McDonald y Siegel, 1985; McGrath, 1999; Micán-Rincón, Acosta-Ortiz y Sánchez-Muñoz, 2015; Sanchez, 1995; Smit y Trigeorgis, 2012; Trigeorgis, 1993; Trigeorgis, 1996; M. G. Urzúa y Cancino, 2016; M. I. G. Urzúa y Escalona, 2017). Aplicaciones más específicas incluyen ejemplos relacionados con decisiones de inversión en tecnología (Benaroch, 2002; Kumar, 2002; McGrath, 1997; Schwartz y Zozaya-Gorostiza, 2003), investigación y desarrollo (Eckhause, Hughes y Gabriel, 2009; Huchzermeier y Loch, 2001; Paxson, 2001; Schneider et al., 2008; Vedovoto y Prior, 2015), patentes (Vasseur y Pérez, 2016), producción y distribución (Cucchiella y Gastaldi, 2006; Cuervo y Botero, 2014; Gamba y Fusari, 2009; Kogut y Kulatilaka, 1994; Tang y Nurmaya Musa, 2011; M. G. Urzúa y Sepúlveda, 2014; Vasseur, Aguirre y Gómez, 2016; Xu, Lu y Li, 2012), así como responsabilidad social corporativa y gestión de riesgos (Cruz, 2002; Husted, 2005; Pineiro-Chousa y Vizcaíno-González, 2016; Pineiro-Chousa, Vizcaíno-González, López-Cabarcos y Romero-Castro, 2017; Vizcaíno-González, 2010).

\section{METODOLOGÍA}

La construcción y puesta en marcha de un campo de golf es un proceso complejo, que se ve afectado por multitud de factores de muy diversa índole, tanto internos como externos. En particular, deben ser considerados elementos relacionados con el uso y sellado del suelo (Díaz, Pedraza y Morales, 2017), parámetros de valoración urbanística (Carmona y Tabales, 2017), así como cuestiones ambientales (Espejo Marín, 2004; Espejo Marín y Cànoves Valiente, 2011; Ligero y Sánchez, 2014) y relativas a la gestión del paisaje (Rodríguez y Corredera, 2017). También deben tenerse en cuenta los hábitos y patrones de comportamiento del público objetivo (Serrano Gómez, 2015), y su reacción ante la calidad de las iniciativas 
comunicativas (García-Tascón y Pradas-García, 2016), así como el tratamiento por parte de los medios (Fernández y Pastor, 2016). Esta enumeración, que en absoluta pretende ser exhaustiva, ilustra la dificultad asociada a la valoración de este tipo de iniciativas. Además, la mutabilidad de muchos de estos factores los hace susceptibles de provocar cambios en las condiciones económicas y financieras de la actividad, y no solo en la etapa de construcción, sino también durante la fase de explotación, dotando de una gran incertidumbre al proyecto. Este es precisamente el motivo por el cual entendemos que la metodología de opciones reales puede resultar apropiada, pues permitiría incorporar la flexibilidad necesaria en la toma de decisiones para reaccionar adecuadamente a dicha incertidumbre. Por ello, el objetivo del artículo es ilustrar la utilidad de esta metodología en este contexto. Y para conseguir dicho objetivo es preciso hacer ciertas simplificaciones didácticas en el planteamiento inicial del proyecto, con la finalidad de centrar la atención del análisis en la propia metodología y su utilidad.

Los datos fueron recopilados a través de entrevistas con directivos y expertos en la construcción, gestión y explotación de campos de golf en diversas zonas de España, así como de otras empresas especializadas en el mundo del golf y el turismo relacionado con el mismo. Estos expertos tienen dilatada experiencia en el sector, y a lo largo de su trayectoria profesional han impulsado proyectos similares al que se presenta en este artículo. Además, son perfectos conocedores de la zona objeto de actuación. De este modo, los expertos hablan desde su propia experiencia y desde su propio conocimiento, así como desde los datos recabados de diversos informes procedentes de la Real Federación Española de Golf, Federaciones Territoriales, y diversas asociaciones turísticas españolas en las que el golf representa una parte importante su actividad.

Se considera el caso de una sociedad promotora que prevé construir y explotar en una finca de su propiedad un campo de golf de 18 hoyos, con casa club, parking, piscina y pistas de pádel. El terreno para la construcción del campo de golf mide 60 hectáreas $(600.000$ metros cuadrados), estimándose un valor para el mismo de 1.980.000 euros. La localización del terreno se asienta en una zona eminentemente turística de las Rías Baixas. Se trata de un entorno caracterizado por un clima atlántico, con un nivel pluviométrico medio-alto, y temperaturas suaves. Dispone de unas excelentes comunicaciones por autopista, con el añadido de la cercanía a Portugal. Además, en la zona y alrededores no se cuenta con ningún campo de golf de 18 hoyos. Por ello, dada la situación geográfica, demográfica y dotacional, se dan razones suficientes para considerar la realización del proyecto.

El proyecto tiene en cuenta la normativa aplicable. En particular, la ley del suelo y el plan de ordenación municipal que le resultan de aplicación. Asimismo, también se contemplan las implicaciones fiscales, que incluyen la consideración de los impuestos sobre el valor añadido (IVA), de bienes inmuebles (IBI), y de sociedades (IS).

Siguiendo estudios de similares características, se establece que el horizonte temporal del estudio sea 30 años, comenzando en el año 2017 como año 0 del proyecto (Gobierno de Canarias, 2015). Se estima que el proyecto planteado obtendrá unos ingresos que, desde el año 2020 (momento en que el campo de golf estará completamente operativo), aumentarán progresivamente en un $4 \%$ anual. Asimismo, en cuanto a los costes, el incremento será de un $2 \%$ anual. La sociedad explotará las instalaciones mediante el sistema de abonados anuales (con cuotas mensuales), sin coste de entrada, de modo que estos abonados en ningún momento serán propietarios de las instalaciones. 
El terreno antes mencionado es aportado por la sociedad, forma parte de sus fondos propios, por lo que no es necesaria su compra. La sociedad promotora financiará el resto de la inversión mediante un préstamo bancario a 6 años, cuyo valor nominal es de 7,8 millones de euros, con un tipo de interés fijo del 4,5\%.

Con estos y otros datos facilitados por los expertos se estiman las cifras relevantes, lo que incluye los costes de inversión, los ingresos y gastos de explotación, las amortizaciones, y el coste de los recursos propios, entre otras. Todo ello con el propósito de calcular el valor actual neto (VAN) del proyecto. El resultado del VAN es de -4.756.230,07 euros, lo que indicaría que el proyecto daría lugar a pérdidas, si se toma únicamente este valor como referencia. Pero dada la elevada incertidumbre del entorno, acrecentada por un horizonte de análisis muy largo, así como la flexibilidad que se puede incorporar al proyecto planteado, se propone la utilización de la metodología de opciones reales como complemento al cálculo del VAN, lo que permitiría plantear y analizar distintos escenarios. Las cinco opciones que se van a analizar son las siguientes:

- Opción de diferir: se plantea la opción de diferir el inicio del proyecto hasta el año 2020, en el cual se celebrarán unos Juegos Olímpicos, siendo el golf parte del programa de dicho evento.

- Opción de abandono: se plantea la posibilidad de desistir en la realización del proyecto y vender el terreno.

- Opción de expansión: se plantea la opción de crear un hotel de cuatro estrellas dentro del complejo.

- Opción de reducción: se plantea la opción de externalizar la gestión de la piscina incluida en el proyecto.

- Opción de elección: se plantea la opción de considerar simultáneamente las opciones de abandonar, expandir y reducir anteriormente descritas.

\section{RESULTADOS}

En primer lugar, se valora la opción de diferir el inicio del proyecto del año 2017 al año 2020, momento en el cual la incertidumbre acerca del efecto de los Juegos Olímpicos quedará resuelta. En los cálculos iniciales se asumió un crecimiento anual de los ingresos del $4 \%$. Ahora se considerarán dos escenarios: un caso optimista, en el que el incremento de los clientes y de sus ingresos sea de un $8 \%$ anual; y un caso pesimista, en el que el incremento sea del $0 \%$, es decir, no existe crecimiento. Se supondrá una probabilidad del $50 \%$ de que ocurra cada uno de los escenarios. En cada escenario optimista y pesimista, se plantea la decisión como la búsqueda del máximo valor entre los resultados de ejecutar o no ejecutar la opción, teniendo en cuenta que hay que capitalizar el valor del desembolso inicial hasta el año 2020. Se utiliza como tasa de capitalización la tasa libre de riesgo. Se toma como referencia la media del bono español a 10 años de los últimos 3 años $(2013$, 2014, 2015), según datos del Banco de España. Esta tasa libre de riesgo es de un $2,54 \%$. Así pues, el valor capitalizado del desembolso inicial es: $9.769 .410,13 *(1+2,54 \%)^{3}=10.532 .907,77$. 
El resultado en ambos escenarios es el siguiente:

- Resultado del VAN en el escenario optimista: 7.411.614,47 $\left(V_{20}^{u} \cong 7,41\right)$

- Resultado del VAN en el escenario pesimista: -11.118.903,24. Por lo tanto, no se realizaría el proyecto $\left(V_{20}^{d}=0\right)$

Asignando una probabilidad del 50\% a cada escenario, el valor del proyecto con la opción de diferir incluida es de 3,44 millones de euros (VAN expandido). Este resultado es muy diferente al obtenido mediante el cálculo del VAN inicial del proyecto (VAN básico). Se puede obtener el valor de la opción de diferir como la diferencia entre el VAN expandido calculado y el VAN básico: $3,44-(-4,76)=8,18$.

El resultado aconseja ejecutar la opción de diferir y, en consecuencia, acometer el proyecto difiriendo su inicio 3 años respecto al plan inicial. Así pues, el resto de las opciones se considerarán sobre el VAN expandido del proyecto por la opción de diferir. Se puede decir, por tanto, que dichas opciones se "encadenan" al proyecto a continuación de la opción de diferir. Para la valoración de las siguientes opciones se utilizará el modelo binomial (Pindado, 2012), estableciendo los siguientes parámetros:

- El vencimiento de las opciones es a 30 años y se asume una periodicidad anual.

- Se utiliza la tasa libre de riesgo anteriormente citada: $2,54 \%$.

- La volatilidad estimada es de un 30\% (Schubert \& Barenbaum, 2007).

- El valor del coeficiente $u$ es 1,3499

- El valor del coeficiente $d$ es 0,7408

- El valor de la probabilidad $p$ es 0,4678

- El precio del subyacente es el VAN del proyecto, expandido con la opción de diferir: 3,44 millones de euros.

La opción de abandono se corresponde con una opción de venta americana, cuyo precio de ejercicio sería el valor de liquidación del proyecto. En el proyecto planteado, este valor se corresponde con el importe que se obtendría al vender el campo de golf, el cual únicamente sería igual al valor del terreno en el año 0 , es decir, 1,98 millones de euros. Con estos datos, se construyen los tres árboles binomiales, cuyo extremo superior izquierdo se presenta de forma combinada.

Se obtiene un valor de 3,87 millones de euros, indicando que la decisión final de la sociedad es la de continuar con el proyecto, es decir, no ejercer la opción de abandono por un valor de 1,98 millones de euros. El valor de la opción de abandono es el resultado de sustraer a este valor expandido el VAN básico de 3,44 millones de euros: 3,87 - 3,44 =0,43.

La opción de expansión es una opción de compra americana, cuyo precio de ejercicio es el coste de la expansión. Se plantea la construcción dentro del complejo de un hotel de cuatro estrellas, lo que supone un coste de 13,5 millones de euros (TINSA, 2014). Se estima el factor de expansión, que indica en cuánto aumentaría la escala del proyecto (sobre los flujos de fondos del proyecto original). Se considera que la cifra de negocio crecería en un $15 \%$ gracias a la construcción de dicho hotel, por lo que el factor de expansión sería de un 1,15. Como en la opción de abandono, se construyen tres árboles combinados del proyecto. 


\section{Figura 1}

\section{ÁRBOL BINOMIAL DE LA OPCIÓN DE ABANDONO}

\begin{tabular}{|c|c|c|c|c|c|}
\hline PERIOODOS & 0 & 1 & 2 & 3 & 4 \\
\hline Evolución del subyacente: & 3,44 & 4,64 & 6,26 & 8,45 & 11,41 \\
\hline Evolución del VA expandido: & 3,87 & 4,97 & 6,51 & 8,63 & 11,53 \\
\hline \multirow[t]{10}{*}{ Decisión } & Continuar & Continuar & Continuar & Continuar & Continuar \\
\hline & & 2,55 & 3,44 & 4,64 & 6,26 \\
\hline & & 3,08 & 3,86 & 4,96 & 6,50 \\
\hline & & Continuar & Continuar & Continuar & Continuar \\
\hline & & & 1,89 & 2,55 & 3,44 \\
\hline & & & 2,55 & 3,08 & 3,85 \\
\hline & & & Continuar & Continuar & Continuar \\
\hline & & & & 1,40 & 1,89 \\
\hline & & & & 2,22 & 2,55 \\
\hline & & & & Continuar & Continuar \\
\hline
\end{tabular}

Figura 2

ÁRBOL BINOMIAL DE LA OPCIÓN DE EXPANSIÓN

\begin{tabular}{|c|c|c|c|c|c|}
\hline PERÍODOS & 0 & 1 & 2 & 3 & 4 \\
\hline Evolución del subyacente: & 3,44 & 4,64 & 6,26 & 8,45 & 11,41 \\
\hline Evolución del VA expandido: & 3,45 & 4,66 & 6,29 & 8,51 & 11,52 \\
\hline \multirow[t]{10}{*}{ Decisión } & \multirow[t]{10}{*}{ No expandir } & No expandir & No expandir & No expandir & No expandir \\
\hline & & 2,55 & 3,44 & \multirow{3}{*}{\begin{tabular}{|l|}
4,64 \\
4,65 \\
\end{tabular}} & 6,26 \\
\hline & & 2,55 & 3,44 & & 6,28 \\
\hline & & \multirow[t]{7}{*}{ No expandir } & No expandir & & No expandir \\
\hline & & & 1,89 & 2,55 & 3,44 \\
\hline & & & 1,89 & 2,55 & 3,44 \\
\hline & & & No expandir & - No expandir & No expandir \\
\hline & & & & 1,40 & 1,89 \\
\hline & & & & 1,40 & 1,89 \\
\hline & & & & No expandir & No expandir \\
\hline
\end{tabular}

Se obtiene un valor de 3,45 millones de euros, indicando que la decisión final de la sociedad es la de continuar con el proyecto inicial, es decir, no ejercer la opción de expansión que suponía la construcción de un hotel. El valor de la opción de expansión es el resultado de sustraer a este valor expandido el VAN básico de 3,44 millones de euros: $3,45-3,44=0,01$.

La opción de reducción es una opción de venta americana, cuyo precio de ejercicio es el ahorro en costes que se obtiene cuando se reduce la dimensión del proyecto. Se plantea la posibilidad de externalizar una de las instalaciones del complejo: la piscina. De este modo, se alquilaría a una empresa externa la explotación de la misma, lo que supondría una serie de ahorros adicionales, lo que incluye gastos de mantenimiento y personal. La suma de todos estos ahorros es igual a 0,804 millones de euros. Se determina el factor de reducción, que indica en cuánto disminuiría la escala del proyecto. Se estima que la pérdida en la cifra de negocio sería de un 1\%. Así pues, el factor de reducción sería de 0,99. Se construyen los tres árboles combinados del proyecto. 
Figura 3

ÁRBOL BINOMIAL DE LA OPCIÓN DE REDUCCIÓN

\begin{tabular}{|c|c|c|c|c|c|}
\hline PERÍODOS & 0 & 1 & 2 & 3 & 4 \\
\hline Evolución del subyacente: & 3,44 & 4,64 & 6,26 & 8,45 & 11,41 \\
\hline Evolución del VA expandido: & 4,21 & 5,40 & 7,00 & 9,17 & 12,10 \\
\hline \multirow[t]{10}{*}{ Decisión } & \multirow[t]{10}{*}{ Reducir } & Reducir & Reducir & Reducir & Reducir \\
\hline & & 2,55 & 3,44 & 4,64 & 6,26 \\
\hline & & 3,32 & 4,21 & 5,40 & 7,00 \\
\hline & & Reducir & Reducir & Reducir & Reducir \\
\hline & & & 1,89 & 2,55 & 3,44 \\
\hline & & & 2,67 & 3,32 & 4,21 \\
\hline & & & Reducir & Reducir & Reducir \\
\hline & & & & 1,40 & 1,89 \\
\hline & & & & 2,19 & 2,67 \\
\hline & & & & Reducir & Reducir \\
\hline
\end{tabular}

Se obtiene un valor de 4,21 millones de euros, lo que da lugar a ejercer la opción. Ello quiere decir que se deja de explotar la piscina y se cede a una empresa externa que se encargará de ello. El valor de la opción de reducción es el resultado de sustraer a este valor expandido el VAN básico de 3,44 millones de euros: 4,21 - 3,44 =0,77.

Una vez analizada de forma independiente cada opción, se plantea un análisis simultáneo de las opciones de abandonar, expandir y reducir, lo que da lugar a una opción de elección. Esta opción permite observar el comportamiento de exclusión que tienen las opciones entre sí. Por ejemplo, para un mismo nodo no puede surgir la opción de ampliación y abandono al mismo tiempo. Se asume que la opción es americana, y el precio de ejercicio es la combinación de los precios de las tres opciones anteriores, teniendo en cuenta también el factor de expansión y el de reducción.

\section{Figura 4}

\section{ÁRBOL BINOMIAL DE LA OPCIÓN DE ELECCIÓN}

\begin{tabular}{|c|c|c|c|c|c|}
\hline PERÍODOS & 0 & 1 & 2 & 3 & 4 \\
\hline Evolución del subyacente: & 3,44 & 4,64 & 6,26 & 8,45 & 11,41 \\
\hline Evolución del VA expandido: & 4,21 & 5,40 & 7,03 & 9,27 & 12,33 \\
\hline \multirow[t]{10}{*}{ Decisión } & Reducir & Reducir & Continuar & Continuar & Continuar \\
\hline & & 2,55 & 3,44 & 4,64 & 6,26 \\
\hline & & 3,33 & 4,21 & 5,40 & 7,02 \\
\hline & & Continuar & Reducir & Reducir & Continuar \\
\hline & & & 1,89 & 2,55 & 3,44 \\
\hline & & & 2,73 & 3,33 & 4,21 \\
\hline & & & Continuar & Continuar & Reducir \\
\hline & & & & 1,40 & 1,89 \\
\hline & & & & 2,33 & 2,73 \\
\hline & & & & Continuar & Continuar \\
\hline
\end{tabular}

Se obtiene un valor de 4,21 millones de euros, siendo la recomendación ejercer la opción de reducción. El valor de la opción de elección es el resultado de sustraer a este valor expandido el VAN básico de 3,44 millones de euros: 4,21 - 3,44 =0,77. 


\section{CONCLUSIONES}

Este estudio analiza la aplicación de la metodología de opciones reales a un proyecto de turismo deportivo: la construcción de un campo de golf. Como punto de partida se calcula el VAN básico del proyecto, que resulta negativo. Si se toma como única referencia, el proyecto no debería ser realizado. Como complemento, se plantea un análisis basado en opciones reales. Se comienza por considerar la opción de diferir el inicio del proyecto tres años, hasta el año 2020, en el cual se celebrarán unos Juegos Olímpicos que incluyen el golf en su programa. El resultado sugiere ejercitar la opción de diferir e iniciar el proyecto en el año 2020 .

Una vez diferido el inicio del proyecto, se plantean a continuación el resto de opciones. La primera de ellas considera la posibilidad de abandonar el proyecto, y debido a la reducida compensación que se ofrece, el resultado sugiere que no debe ejercerse. La siguiente opción considera la posibilidad de expansión del proyecto mediante la construcción de un hotel de cuatro estrellas, lo que resulta un gasto demasiado elevado en comparación con el aumento de la cifra de negocio que ello supondría, por lo que dicha opción tampoco debe ejercerse. Además, se considera la opción de reducción mediante el alquiler de la explotación de la piscina a una empresa externa, lo que implica un gran ahorro a lo largo de la vida del proyecto, con una pérdida mínima en la cifra de negocio, gracias a lo cual se aconseja ejercer la opción de reducción. Por último, se considera la opción de elección que tiene en cuenta la interacción entre las opciones de abandono, expansión y reducción, así como su carácter de mutua exclusión, concluyendo que la opción de reducción debe ejercerse.

Por tanto, tras el análisis realizado se sugiere diferir el inicio del proyecto hasta el año 2020, y ejecutarlo entonces con una reducción en la dimensión inicialmente diseñada para el mismo, que pasa por externalizar la gestión de la piscina incluida en el complejo deportivo. Es decir, se ejercen las opciones de diferir y reducir el proyecto de forma encadenada. En el siguiente esquema se resume la valoración del proyecto de inversión

Figura 5

RESUMEN DE LAS OPCIONES VALORADAS

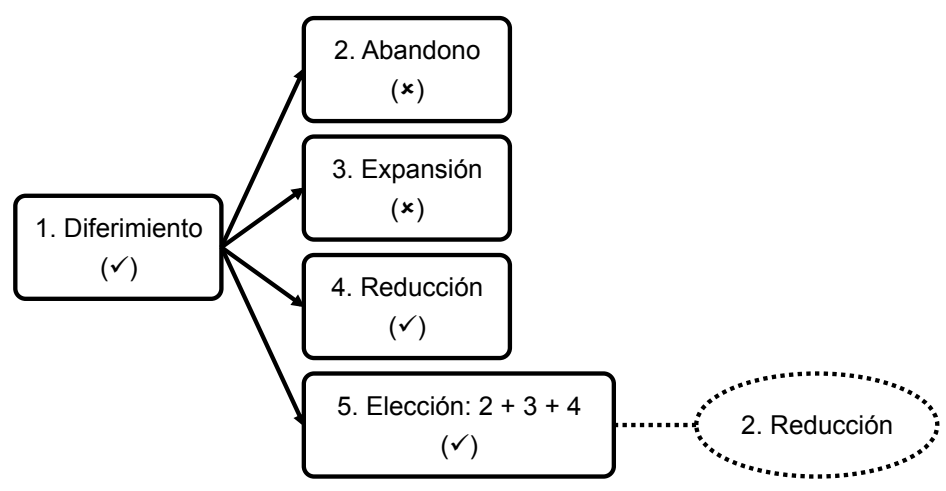


La puesta en funcionamiento de un campo de golf es un proceso muy complejo, condicionado por muchos y diversos factores, que pueden afectar a la viabilidad económicafinanciera del proyecto a lo largo de toda su vida. La metodología de opciones reales aporta la flexibilidad necesaria para reaccionar a esos cambios durante el desarrollo del proyecto. Por ello, el objetivo del artículo ha sido ilustrar con un ejemplo numérico dicho potencial metodológico, para lo cual ha sido necesario asumir ciertas simplificaciones en las condiciones iniciales del proyecto, que exigirían un análisis exhaustivo, lo que excede al propósito del artículo.

En última instancia, y generalizando las conclusiones, el estudio demuestra la utilidad de la metodología de opciones reales para valorar proyectos de turismo deportivo. Debido a su dimensión temporal y financiera, las decisiones acerca de estos proyectos no deben ser consideradas del tipo "ahora o nunca", lo que es un enfoque inherente a los métodos clásicos de valoración. Si el entorno se caracteriza por una elevada incertidumbre, y si es posible precisar la existencia de oportunidades para un desarrollo flexible o modular del proyecto, la metodología de opciones reales propicia un análisis más realista.

\section{REFERENCIAS}

ABEL, A.B., DIXIT, A.K., EBERLY, J.C. y PINDYCK, R.S. (1996): «Options, the value of capital, and investment», The quarterly Journal of economics, $\mathrm{n}^{\circ} 111$, pp. 753-777.

AMRAM, M. y KULATILAKA, N. (1999): Real options: Managing strategic investment in an uncertain world. Boston, Harvard Business School Press.

AYÚS, T., LENIN, A. y ARISTIZÁBAL VELÁSQUEZ, R.E. (2012): «Las opciones reales como metodología alternativa en la evaluación de proyectos de inversión», Ecos de Economía, n ${ }^{\circ} 16$, pp. 29-44.

BENAROCH, M. (2002): «Managing information technology investment risk: A real options perspective», Journal of Management Information Systems, $\mathrm{n}^{\circ}$ 19, pp. 43-84.

BOWMAN, E.H. y HURRY, D. (1993): «Strategy through the option lens: An integrated view of resource investments and the incremental-choice process», Academy of management review, $\mathrm{n}^{\circ} 18$, pp. 760-782.

BRENNAN, M.J. y SCHWARTZ, E.S. (1985): «Evaluating natural resource investments», Journal of Business, ${ }^{\circ}$ 58, pp. 135-157.

CARMONA, F.J.R. y TABALES, J.N. (2017): «Alternativas para la valoración de inmuebles urbanos», Revista Publicando, n 4, pp. 3-19.

COPELAND THOMAS, KOLLER, T. y MURRIN, J. (1994): Valuation: measuring and managing the value of companies. New Jersey, Wiley.

COPELAND, T. y ANTIKAROV, V. (2001): Real options. New York, Texere.

CRUZ, M.G. (2002): Modeling, measuring and hedging operational risk. New York, John Wiley \& Sons.

CUCCHIELLA, F. y GASTALDI, M. (2006): «Risk management in supply chain: a real option approach», Journal of Manufacturing Technology Management, $\mathrm{n}^{\circ} 17, \mathrm{pp}$. 700-720.

CUERVO, F.I. y BOTERO, S.B. (2014): «Aplicación de las opciones reales en la toma de decisiones en los mercados de electricidad», Estudios Gerenciales, n 30, pp. 397-407. 
DÍAZ, A.R., PEDRAZA, A.C. y MORALES, A.P. (2017): «Expansión urbana y turismo en la Comarca del Campo de Cartagena-Mar Menor (Murcia). Impacto en el sellado del suelo», Cuadernos de Turismo, $\mathrm{n}^{\circ} 39$, pp. 521-546.

DIXIT, A.K. (1994): Investment under uncertainty. New Jersey, Princeton university press.

ECKHAUSE, J.M., HUGHES, D.R. y GABRIEL, S.A. (2009): «Evaluating real options for mitigating technical risk in public sector R\&D acquisitions», International Journal of Project Management, $\mathrm{n}^{\circ}$ 27, pp. 365-377.

ESPEJO MARÍN, C. (2004): «Campos de golf y medio ambiente. Una interacción necesaria», Cuadernos de Turismo, $\mathrm{n}^{\circ} 14$, pp. 67-111.

ESPEJO MARÍN, C. y CÀNOVES VALIENTE, G. (2011): «Política de usos del agua en los campos de golf de España», Documents d>anàlisi geogràfica, n ${ }^{\circ}$ 57, pp. 255-277.

FERNÁNDEZ, J.G.F. y PASTOR, E.M. (2016): «El tratamiento informativo y publicitario de los diarios digitales especializados en golf en España, Cuadernos de Turismo, $\mathrm{n}^{\circ}$ 37, pp. 157-174.

GAMBA, A. y FUSARI, N. (2009): «Valuing modularity as a real option», Management Science, $\mathrm{n}^{\circ} 55$, pp. 1.877-1.896.

GARCÍA MACHADO, J.J. (2001): Opciones reales: aplicaciones de la teoría de opciones a las finanzas empresariales. Madrid, Piramide.

GARCÍA-TASCÓN, M. y PRADAS-GARCÍA, M. (2016): «i La transparencia de las páginas web ayudan en la captación de clientes? Análisis de los campos de golf en Andalucía», Intangible Capital, n 12, pp. 805-821.

GOBIERNO DE CANARIAS (2015): Informe de Valoración Campo de Golf en San Bartolomé de Tirajana. Las Palmas de Gran Canaria, Gobierno de Canarias.

GONZÁLEZ-ECHEVERRI, G., MORA-VALENCIA, A. \& SOLANO, J.G. (2015): «Opciones reales aplicadas en redes integradas de servicios de salud empleando diferentes métodos de estimación de la volatilidad», Estudios Gerenciales, n 31, pp. 287-298.

HUCHZERMEIER, A. y LOCH, C.H. (2001): «Project Management Under Risk: Using the Real Options Approach to Evaluate Flexibility in R... D», Management Science, $\mathrm{n}^{\circ} 47$, pp. 85-101.

HUSTED, B.W. (2005): «Risk management, real options, corporate social responsibility», Journal of Business Ethics, n 60, pp. 175-183.

KOGUT, B. y KULATILAKA, N. (1994): «Operating flexibility, global manufacturing, and the option value of a multinational network», Management Science, $\mathrm{n}^{\circ}$ 40, pp. 123-139.

KOLLER, T., GOEDHART, M. y WESSELS, D. (2010): Valuation: measuring and managing the value of companies. New Jersey, John Wiley \& Sons.

KUMAR, R.L. (2002): «Managing risks in IT projects: an options perspective», Information \& Management, $\mathrm{n}^{\circ} 40, \mathrm{pp} .63-74$.

LIGERO, F.J.R. y SÁNCHEZ, A.V. (2014): «La legitimidad social de los campos de golf andaluces mediante prácticas ambientales y su relación con el desempeño: un estudio longitudinal», Revista Galega de Economía, n 23, pp. 5-26.

MASCAREÑAS, J. (2010): Finanzas Corporativas. Madrid, Universidad Complutense de Madrid. 
MCDONALD, R.L. y SIEGEL, D.R. (1985): «Investment and the valuation of firms when there is an option to shut down», International Economic Review, $\mathrm{n}^{\circ}$ 26, pp. 331-349.

MCGRATH, R.G. (1997): «A real options logic for initiating technology positioning investments», Academy of Management Review, n 22, pp. 974-996.

MCGRATH, R.G. (1999): «Falling forward: Real options reasoning and entrepreneurial failure», Academy of Management Review, $\mathrm{n}^{\circ}$ 24, pp. 13-30.

MICÁN-RINCÓN, C.A., ACOSTA-ORTIZ, P.Y. y SÁNCHEZ-MUÑOZ, A.F. (2015): «Evaluación de un proyecto estratégico de administración de capacidad considerando flexibilidad operativa y opciones reales», Entramado, n 11, pp. 226-239.

MUN, J. (2002): Real options analysis: Tools and techniques for valuing strategic investments and decisions. Nueva Jersey, John Wiley \& Sons.

PAXSON, D.A. (2001): «Introduction to real R\&D options», $R$ and D Management, $\mathrm{n}^{\circ}$ 31 , pp. 109-114.

PINDADO, J. (2012): Finanzas empresariales. Madrid, Paraninfo.

PINEIRO-CHOUSA, J. y VIZCAÍNO-GONZÁLEZ, M. (2016): «A quantum derivation of a reputational risk premium», International Review of Financial Analysis, $\mathrm{n}^{\circ} 47$, pp. 304-309. doi:10.1016/j.irfa.2016.01.003

PINEIRO-CHOUSA, J., VIZCAÍNO-GONZÁLEZ, M., LÓPEZ-CABARCOS, M.Á у ROMERO-CASTRO, N. (2017): «Managing Reputational Risk through Environmental Management and Reporting: An Options Theory Approach», Sustainability, $\mathrm{n}^{\circ}$ 9, pp. 376.

RODRÍGUEZ, M.F.M. y CORREDERA, S.R. (2017): «La costa occidental de la provincia de Málaga: rasgos generales de su paisaje», PH: Boletín del Instituto Andaluz del Patrimonio Histórico, $\mathrm{n}^{\mathrm{o}} 25$, pp. 28-41.

SÁNCHEZ, R. (1995): «Strategic flexibility in product competition», Strategic Management Journal, $\mathrm{n}^{\circ} 16$, pp. 135-159.

SCHNEIDER, M., TEJEDA, M., DONDI, G., HERZOG, F., KEEL, S. y GEERING, H. (2008): «Making real options work for practitioners: a generic model for valuing R\&D projects», R\&d Management, $\mathrm{n}^{\circ} 38, \mathrm{pp} .85-106$.

SCHWARTZ, E.S. y ZOZAYA-GOROSTIZA, C. (2003): «Investment under uncertainty in information technology: acquisition and development projects», Management Science, $\mathrm{n}^{\circ} 49$, pp. 57-70.

SERRANO GÓMEZ, V. (2015): «Evolución de la práctica del golf, caracteristicas y dimensión económica», Revista Española de Educación Física y Deportes, pp. 63-80.

SMIT, H.T. y TRIGEORGIS, L. (2012): Strategic investment: Real options and games. Princeton, Princeton University Press.

TANG, O. y NURMAYA MUSA, S. (2011): «Identifying risk issues and research advancements in supply chain risk management», International Journal of Production Economics, $\mathrm{n}^{\circ} 133$, pp. 25-34.

TRIGEORGIS, L. (1993): «Real options and interactions with financial flexibility», Financial Management, $\mathrm{n}^{\circ} 22$, pp. 202-224.

TRIGEORGIS, L. (1996): Real Options: Managerial flexibility and Strategy Inresource Allocation. USA, The MIT Press. 
URZÚA, M.G. y CANCINO, J.H. (2016): «Valoración de una empresa de agua potable mediante opciones reales», Industrial Data, no 19, pp. 30-41.

URZÚA, M.G. y SEPÚLVEDA, J.G. (2014): «Opciones reales implícitas en una empresa distribuidora de alimentos», Industrial Data, n 17, pp. 39-47.

URZÚA, M.I.G. y ESCALONA, K.J.M. (2017): «Nuevos enfoques de valoración de empresas. Aplicación de la metodología de opciones a una empresa de transporte de carga chilena», Horizontes Empresariales, no 15, pp. 30-44.

VASSEUR, J.P., AGUIRRE, M.M. y GÓMEZ, M.G. (2016): «Evaluación mediante opciones reales de proyectos de inversión en el sector de distribución de combustibles», Estocástica: Finanzas y riesgo, $\mathrm{n}^{\circ}$ 6, pp. 219-246.

VASSEUR, J.P. y PÉREZ, C.C. (2016): «Valoración de patentes farmacéuticas a través de opciones reales: equivalentes de certeza y función de utilidad», Contaduría y Administración, $\mathrm{n}^{\circ}$ 61, pp. 794-814.

VEDOVOTO, G.L. y PRIOR, D. (2015): «Opciones reales: una propuesta para valorar proyectos de $\mathrm{I}+\mathrm{D}$ en centros públicos de investigación agraria», Contaduría y Administración, ${ }^{\circ} 60$, pp. 145-179.

VIZCAÍNO-GONZÁLEZ, M. (2010): «Riesgo de reputación: revision teórica y aproximación a su valoración», Revista Galega de Economía, nº 19, pp. 199-216.

XU, S.X., LU, Q. y LI, Z. (2012): «Optimal modular production strategies under market uncertainty: A real options perspective», International Journal of Production Economics, $\mathrm{n}^{\circ} 139$, pp. 266-274. 